\title{
Directable Fuzzy Automata
}

\author{
V. Karthikeyan \\ Assistant Professor \\ Departent of Mathematics \\ Annamalai University \\ Chidambaram \\ Tamilnadu, India
}

\author{
M. Rajasekar \\ Assistant Professor \\ Mathematics section,FEAT \\ Annamalai University \\ Chidambaram \\ Tamilnadu, India
}

\begin{abstract}
The aim of this paper is testing the directability of a given fuzzy automaton. A fuzzy automaton is directable if there exists a word, a directing word, which takes each state of a fuzzy automaton to a single state with some membership value. In this paper, we proposed a method for testing the directability of a fuzzy automaton using the mergeability relation.
\end{abstract}

\section{Keywords}

Directable, Mergeable, Congruence and Directing Congruence.

\section{INTRODUCTION}

Fuzzy set is a generalization of a classical set was introduced by Zadeh in 1965 [11]. This concept is applied in different discipline including medical sciences, artificial intelligence, pattern recognition and automata theory. Fuzzy ideas applied in automata was first proposed by Wee in 1967 [10]. Santos proposed fuzzy automata as a model of pattern recognition [9]. J. N. Mordeson and D. S. Malik gave a detailed account of fuzzy automata and applications in their book 2002 [8].

A fuzzy automaton is directable if there exists a word, a directing word, which takes each state of a fuzzy automaton to a single state with some membership value.

Using the concept of fuzzy recognizer, word recognized by fuzzy recognizer and the language recognized by fuzzy recognizer we prove that for any directable fuzzy automaton, the set of all directing words of a fuzzy automaton belongs to the language recognized by fuzzy recognizer.

We provide a necessary and sufficient condition for a fuzzy automaton to be directable, congruence of a fuzzy automaton to be directing. Further, we proposed a method for testing the directability of a fuzzy automaton using the mergeability relation.

Finally, We proposed an algorithm to find whether a fuzzy automaton is directable or not, using the mergeability relation of the states with example.

\section{PRELIMINARIES}

Let $X$ denote a universal set. Then a fuzzy set $A$ in $X$ is set of ordered pairs: $A=\left\{\left(x, \mu_{A}(x) \mid x \in X\right\}, \mu_{A}(x)\right.$ is called the membership function or grade of membership of $x$ in $A$ which maps $X$ to the membership space $[0,1][12$.
A finite fuzzy automaton is a system of 5 tuples, $M=\left(Q, \Sigma, f_{M}, q_{0}, F\right)$,

where, $Q$ is set of states, $\Sigma$ is input symbols, $f_{M}$ is transition function from $Q \times \Sigma \times Q \rightarrow[0,1], q_{0}$ is an initial state and $q_{0} \in Q$, and $F \subseteq Q$ set of final states. The transition in a fuzzy automaton is as follows:

$f_{M}\left(q_{i}, a, q_{j}\right)=\mu, 0 \leq \mu \leq 1$, means that when $M$ is in state $q_{i}$ and reads the input $a$ will move to the state $q_{j}$ with weight function $\mu$.

$f_{M}$ can be extended to $Q \times \Sigma^{*} \times Q \rightarrow[0,1]$ by,

$$
\begin{gathered}
f_{M}\left(q_{i}, \epsilon, q_{j}\right)= \begin{cases}1 & \text { if } q_{i}=q_{j} \\
0 & \text { if } q_{i} \neq q_{j}\end{cases} \\
f_{M}\left(q_{i}, w, q_{m}\right)=\operatorname{Max}\left\{\operatorname { M i n } \left\{f_{M}\left(q_{i}, a_{1}, q_{1}\right), f_{M}\left(q_{1}, a_{2}, q_{2}\right), \ldots,\right.\right. \\
\left.\left.f_{M}\left(q_{m-1}, a_{m}, q_{m}\right)\right\}\right\}
\end{gathered}
$$

for $w=a_{1} a_{2} a_{3} \ldots a_{m} \in \Sigma^{*}$, where Max is taken over all the paths from $q_{i}$ to $q_{m}$.

We can represent transitions more conveniently by the matrix notation as follows:

For each $a \in \Sigma$, we can form a $n \times n$ matrix $F(a)$ whose $(i, j)^{t h}$ element is $f_{M}\left(q_{i}, a, q_{j}\right)$. Let $w \in \Sigma^{*}$ and $w=a_{1} a_{2} a_{3} \ldots a_{m}$ then $F(w)=F\left(a_{1}\right) \circ F\left(a_{2}\right) \circ \ldots . . \circ F\left(a_{m}\right)$.

In other words, $F(w)$ is the fuzzy sum of fuzzy products of weights taken over the paths in the automaton [4].

Throughout this paper, we consider a fuzzy automaton without initial state and final state and $M$ denotes $M=\left(Q, \Sigma, f_{M}\right), f_{M}$ is transition function from $Q \times \Sigma \times Q \rightarrow[0,1]$.

A fuzzy automaton $M$ is called deterministic if for each $a \in \Sigma$ and $q_{i} \in Q$, there exists a unique state $q_{a}$ such that $f_{M}\left(q_{i}, a, q_{a}\right)>0$ otherwise it is called nondeterministic [3].

Let $M^{\prime}=\left(Q^{\prime}, \Sigma, f_{M^{\prime}}\right), Q^{\prime} \subseteq Q$ and $f_{M^{\prime}}$ is the restriction of $f_{M}$. The fuzzy automaton $M^{\prime}$ is called a subautomaton of $M$ if

(i) $f_{M^{\prime}}: Q^{\prime} \times \Sigma \times Q^{\prime} \rightarrow[0,1]$ and

(ii) For any $q_{i} \in Q^{\prime}$ and $f_{M^{\prime}}\left(q_{i}, u, q_{j}\right)>0$ for some $u \in \Sigma^{*}$, then $q_{j} \in Q^{\prime}$. $M$ is said to be strongly connected if for every $q_{i}, q_{j} \in Q$, there exists $u \in \Sigma^{*}$ such that $f_{M}\left(q_{i}, u, q_{j}\right)>0$. Equivalently, $M$ is strongly connected if it has no proper subautomaton [8].

An equivalence relation $R$ on $Q$ in $M$ is called a congruence relation if for all $q_{i}, q_{j} \in Q$ and $a \in \Sigma, q_{i} R q_{j}$ implies that, then there 
exists $q_{l}, q_{k} \in Q$ such that $f_{M}\left(q_{i}, a, q_{l}\right)>0, f_{M}\left(q_{j}, a, q_{k}\right)>0$ and $q_{l} R q_{k}$, 1, 2].

Let $M$ be a fuzzy automaton. The quotient fuzzy automaton determined by the congruence $\cong$ is a fuzzy automaton $M / \cong=\left(Q / \cong, \Sigma, f_{M / \cong}\right)$, where $Q / \cong=\left\{Q_{i}=\left[q_{i}\right]\right\}$ and $f_{M / \cong}\left(Q_{1}, a, Q_{2}\right)=\operatorname{Min}\left\{f_{M}\left(q_{1}, a, q_{2}\right)>0 / q_{1} \in Q_{1}, q_{2}\right.$

\section{DIRECTABLE FUZZY AUTOMATA AND DIRECTING CONGRUENCES}

Let $M$ be a fuzzy automaton. For every $q_{i} \in Q$, if there exists a state $q_{j} \in Q$ and $u \in \Sigma^{*}$ such that $f_{M}\left(q_{i}, u, q_{j}\right)>0$. In that case, the word $u$ is said to be a directing word of $M$. If $M$ has a directing word, then we say that $M$ is a directable fuzzy automaton. The set of all directing words of $M$ is denoted by $D W(M)$ [6].

We say that two states $q_{i}, q_{j} \in Q$ are said to be mergeable or reducible if there exists a word $u \in \Sigma^{*}$ and $q_{j} \in Q$ such that $f_{M}\left(q_{i}, u, q_{k}\right)>0 \Leftrightarrow f_{M}\left(q_{j}, u, q_{k}\right)>0$ [5].

Let $M$ be a fuzzy automaton. The set of all equivalence relations on a set $Q$ is denoted by $E q(Q)$. Let $\delta_{M} \in E q(Q)$. If any two states $q_{i}, q_{j} \in Q$ is called $\delta_{M}$-Mergeable, then there exists $\left(q_{k}, q_{l}\right) \in \delta_{M}$ such that $f_{M}\left(q_{i}, w, q_{k}\right)>0$ and $f_{M}\left(q_{j}, w, q_{l}\right)>0$, for some $w \in \Sigma^{*}$.

Let $M$ be a fuzzy automaton. Let $\rho$ be the congruence relation on the states set $Q$ in $M$. If $\rho$ is called directing congruence, then the quotient fuzzy automaton $M / \rho$ is a directable fuzzy automaton.

\subsection{Fuzzy Recognizer $[\overline{8}]$}

Let $Q$ and $\Sigma$ be finite subsets. A fuzzy recognizer is a five tuple $M_{1}=\left(Q, \Sigma, f_{M_{1}}, \psi, \xi\right)$, where,

(i) $Q$ is a finite nonempty set of states,

(ii) $\Sigma$ is a finite nonempty set of input symbols,

(iii) $f_{M_{1}}: Q \times \Sigma \times Q \rightarrow[0,1]$ is a function, called the fuzzy transition function,

(iv) $\psi$ is a initial fuzzy subset of $Q$, i.e., $\psi: Q \rightarrow[0,1]$, called the initial fuzzy state, and

(v) $\xi$ is a final fuzzy subset of $Q$, i.e., $\xi: Q \rightarrow[0,1]$, called the fuzzy subset of final states.

\section{Note}

Clearly, if $M_{1}=\left(Q, \Sigma, f_{M_{1}}, \psi, \xi\right)$ is a fuzzy recognizer, then $M=\left(Q, \Sigma, f_{M}\right)$ is a fuzzy automaton. We call $M$ the fuzzy automaton associated with the fuzzy recognizer $M_{1}$.

\subsection{Word Recognized by Fuzzy Recognizer[8]}

Let $M_{1}=\left(Q, \Sigma, f_{M_{1}}, \psi, \xi\right)$ be a fuzzy recognizer. Let $w \in \Sigma^{*}$. Then $w$ is said to be recognized by $M_{1}$ if

$\vee_{q_{i} \in Q}\left(\psi\left(q_{i}\right) \wedge\left(\vee_{q_{j} \in Q}\left\{f_{M_{1}}\left(q_{i}, w, q_{j}\right) \wedge \xi\left(q_{j}\right)\right\}\right)\right)>0$.

\subsection{Language Recognized by Fuzzy Recognizer $[\overline{8}]$}

Let $M_{1}=\left(Q, \Sigma, f_{M_{1}}, \psi, \xi\right)$ be a fuzzy recognizer. Let $L\left(M_{1}\right)=\left\{w \in \Sigma^{*} / w\right.$ is recognized by $\left.M_{1}\right\} . L\left(M_{1}\right)$ is called the language recognized by the fuzzy recognizer $M_{1}$, and the set of all fuzzy recognizable language by $M_{1}$ associated with $M=\left(Q, \Sigma, f_{M}\right)$ is denoted by $F \operatorname{Rec}(M)$.

\section{PROPERTIES OF DIRECTABLE FUZZY AUTOMATA AND DIRECTING CONGRUENCES}

LEMMA 4.1. For any directable fuzzy automaton $M=\left(Q, \Sigma, f_{M}\right), \Sigma^{*} D W(M) \Sigma^{*}=D W(M)$

$Q_{2}$ aprbof: $\left.\Sigma\right\}$ [7].

Since $M$ is a directable fuzzy automaton, there exists a directing word $w \in \Sigma^{*}$. Now, $w \in D W(M) \Rightarrow \lambda w \lambda \in \Sigma^{*} D W(M) \Sigma^{*}$. Therefore, $D W(M) \subseteq \Sigma^{*} D W(M) \Sigma^{*}-(1)$

Take $w_{1} \in \Sigma^{*} D W(M) \Sigma^{*}, w_{1}=u w v, w \in D W(M)$ and $u, v \in$ $\Sigma^{*}$. Since $w$ is a directing word, we have $f_{M}\left(q_{i}, u w v, q_{k}\right)>0$, for all $q_{i} \in Q$, and some $q_{k} \in Q$. Therefore, $w_{1}=u w v$ is also a directing word. Hence, $w_{1} \in D W(M)$.

That is, $\Sigma^{*} D W(M) \Sigma^{*} \subseteq D W(M)-(2)$

From (1) \& (2), $\Sigma^{*} D W(M) \Sigma^{*}=D W(M)$

LEMMA 4.2. For any directable fuzzy automaton $M=\left(Q, \Sigma, f_{M}\right), D W(M) \in F \operatorname{Rec}(M)$

\section{Proof:}

Consider any directable fuzzy automaton, we associate a fuzzy recognizer $M_{d}=\left(2^{Q}, \Sigma, f_{M_{d}}, \psi(Q), \xi(S)\right)$, where $S$ is set of all singleton sets. $\psi(Q)=1$ and $\psi\left(Q_{1}\right)=0, \forall Q_{1} \in 2^{Q} . \xi(S)=1$ for singleton set $S \in 2^{Q}$ and $\xi\left(Q_{2}\right)=0, \forall Q_{2} \in 2^{Q}$. The transition function $f_{M_{d}}$ in $M_{d}$ is defined by

$f_{M_{d}}(P, a, T)=\operatorname{Min}\left\{f_{M}\left(q_{i}, a, q_{j}\right), q_{i} \in P, q_{j} \in T\right\}, P, T \in$ $2^{Q}$ for $a \in \Sigma$.

Clearly, $M_{d}$ is a deterministic fuzzy automaton. Let $w \in$ $\Sigma^{*}$ be a directing word of $M$. That is, $f_{M_{d}}(Q, w, S)=$ $\vee\left\{\psi(Q) \wedge f_{M_{d}}(Q, w, S) \wedge \xi(S)\right\}>0$.

$w$ is recognized by $M_{d}$. Hence, the set of all directing words is a language recognized by the fuzzy automaton $M_{d}$. Hence, $D W(M) \in F \operatorname{Rec}(M)$.

THEOREM 4.1. A fuzzy automaton $M=\left(Q, \Sigma, f_{M}\right)$ is directable if and only if all pairs of states of $Q$ in $M$ are mergeable.

\section{Proof:}

A fuzzy automaton $M$ is directable. Since it is directable, there exists a directing word $u \in \Sigma^{*}$ and $q_{j} \in Q$ such that $f_{M}\left(q_{i}, u, q_{j}\right)>$ 0 , for every $q_{i} \in Q$. Let $q_{k}, q_{l} \in Q$. Then by the hypothesis, we have

$f_{M}\left(q_{k}, u, q_{j}\right)>0 \Leftrightarrow f_{M}\left(q_{l}, u, q_{j}\right)>0$. Therefore, $q_{k}$ and $q_{l}$ are mergeable.

Conversely, Assume that $M$ is not a directable fuzzy automaton. That is, we assume that all states are mergeable in two states $q_{k}$ and $q_{l}$ in $Q$. Then there exists $w_{1} \in \Sigma^{*}$ such that $f_{M}\left(q_{i}, w_{1}, q_{k}\right)>0$ and $f_{M}\left(q_{j}, w_{1}, q_{l}\right)>0$, for $q_{i}^{\prime} s, q_{j}^{\prime} s \in Q$.

Now, take the states $q_{k}$ and $q_{l}$. By the hypothesis, the states $q_{k}$ and $q_{l}$ are mergeable. That is, there exists a word $w_{2} \in \Sigma^{*}$ and $q_{m} \in Q$ such that $f_{M}\left(q_{k}, w_{2}, q_{m}\right)>0 \Leftrightarrow f_{M}\left(q_{l}, w_{2}, q_{m}\right)>0$, for every $q_{k}, q_{l} \in Q$.

Now, $f_{M}\left(q_{i}, w_{1} w_{2}, q_{m}\right)>0$, for every $q_{i} \in Q$, which is a contradiction to our assumption. Hence, $M$ is a directable fuzzy automaton.

THEOREM 4.2. Let $M=\left(Q, \Sigma, f_{M}\right)$ be a fuzzy automaton. A congruence $\rho$ of a fuzzy automaton $M$ is directing if and only if all pairs of states on $Q$ in $M$ are $\rho$-mergeable. 


\section{Proof:}

Assume that congruence $\rho$ of a fuzzy automaton $M$ is directing. By the definition of congruence it has an equivalence class say $Q_{1}=$ $\left\{P_{1}, P_{2} \ldots . P_{n}\right\}$. Now, consider the quotient fuzzy automaton of $M$ say $M / \rho=\left(Q_{1}, \Sigma, f_{M / \rho}\right)$.

Since congruence $\rho$ of $M$ is directing, then there exists a directing word say $w \in \Sigma^{*}$ and $P_{j} \in Q_{1}$ such that $f_{M / \rho}\left(P_{i}, w, P_{j}\right)>0$, for every $P_{i} \in Q_{1}$.

Let $q_{i}, q_{j} \in Q$. Then either $q_{i}, q_{j} \in P_{i}$ or $q_{i} \in P_{i}$ and $q_{j} \in P_{k}$.

Let $q_{i}, q_{j} \in P_{i}$. Then $f_{M}\left(q_{i}, w, q_{k}\right)>0$ and $f_{M}\left(q_{j}, w, q_{l}\right)>0$, for some $q_{k}, q_{l} \in P_{j}$. This implies that $\left(q_{k}, q_{l}\right) \in \rho$.

Now, let $q_{i} \in P_{i}$ and $q_{j} \in P_{k}$, for $i \neq k$ be any two states in $Q$. Then $f_{M}\left(q_{i}, w, q_{m}\right)>0$ and $f_{M}\left(q_{j}, w, q_{n}\right)>0$, for some $\left(q_{m}, q_{n}\right) \in P_{j}$. This implies that $\left(q_{m}, q_{n}\right) \in \rho$. All pairs of states on $Q$ in $M$ are $\rho$-mergeable.

Conversely, assume that all pairs of states on $Q$ in $M$ are $\rho$ mergeable. Since $\rho$ is congruence we can construct the quotient fuzzy automaton say $M / \rho=\left(Q_{1}, \Sigma, f_{M / \rho}\right)$, where, $Q_{1}=$ $\left(P_{1}, P_{2} \ldots P_{n}\right)$.

Assume that in $M / \rho$ all states of $Q_{1}$ are merged in two distinct states $P_{j}$ and $P_{k}$. That is, there exists a word $w_{1} \in \Sigma^{*}$ such that $f_{M / \rho}\left(P_{i}, w_{1}, P_{j}\right)>0$ and $f_{M / \rho}\left(P_{i}, w_{1}, P_{k}\right)>0$, for some $i$.

Let $q_{i} \in P_{j}$ and $q_{j} \in P_{k}$. Since all pairs of $Q$ in $M$ are $\rho$-mergeable, there exists a word $w_{2} \in \Sigma^{*}$ such that $f_{M}\left(q_{i}, w_{2}, q_{r}\right)>0, f_{M}\left(q_{j}, w_{2}, q_{s}\right)>0$, for some $\left(q_{r}, q_{s}\right) \in P_{l}$.

Let $w=w_{1} w_{2} \in \Sigma^{*}$. Then $f_{M / \rho}\left(P_{i}, w, P_{l}\right)>0$, for every $P_{i} \in Q_{1}$

It is a contradiction to our assumption. Hence, $M / \rho$ is a directable fuzzy automaton.

\section{ALGORITHM FOR TESTING DIRECTABILITY}

The algorithm for testing directability contains two auxillary data structures. A $n \times n$ fuzzy matrix $M[i, j]$ and list NewPair of pairs of states.

We assume that $Q=\left\{q_{1}, q_{2}, q_{3} \ldots . q_{n}\right\}$. If the pair $\left(q_{i}, q_{j}\right)$ is mergeable, then $0<M[i, j] \leq 1$. Since it suffices to consider just the pairs $(i, j)$, where $1 \leq i<j \leq n$, we actually need just upper part of $M$.

A pair $\left(q_{i}, q_{j}\right)$ appears in NewPair when $q_{i}$ and $q_{j}$ have found to be mergeable. For finding further mergeable pairs, we use inverted transition table.

The inverted transition table $I=\left(I\left[q_{k}, a\right]\right)$, for any $q_{k} \in Q, a \in \Sigma$ is defined as follows:

$I\left[q_{k}, a\right]=\left\{q_{j} \in Q / f_{M}\left(q_{j}, a, q_{k}\right)>0\right\}$, for any $q_{k} \in Q, a \in \Sigma$.

\section{Algorithm:}

\section{Step1:}

[Initialize $\mathrm{M}$ and NewPair] $M[i, j]=0$ for all $1 \leq i<j \leq n$, and NewPair $=\{\epsilon\}$ (the empty list).

Step2:

Form the inverted transition table I.

Step3:

Find all pairs $\left(q_{k}, a\right) \in Q \times \Sigma$ for which $\left|I\left[q_{k}, a\right]\right|>1$. For every such $\left(q_{k}, a\right)$ consider each pair $q_{i}, q_{j} \in I\left[q_{k}, a\right]$ with $i<j$. If $M[i, j]=0$, let $M[i, j]=\operatorname{Min}\left\{f_{M}\left(q_{i}, w, q_{k}\right), f_{M}\left(q_{j}, w, q_{k}\right)\right\}$

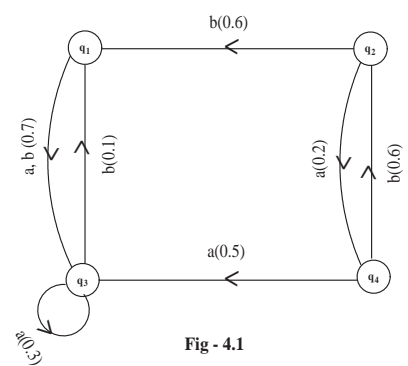

Example

for some $w \in \Sigma^{*}$ and append $\left(q_{i}, q_{j}\right)$ to NewPair.

Step4:

Until NewPair $=\{\epsilon\}$ do the following. Delete the first pair from NewPair. Suppose it $\left(q_{l}, q_{m}\right)$. From $I$ find all pairs $\left(q_{i}, q_{j}\right), i<j$, such that for some $a \in \Sigma$,

$q_{i} \in I\left[q_{l}, a\right]$ and $q_{j} \in I\left[q_{m}, a\right]$, or $q_{i} \in I\left[q_{m}, a\right]$ and $q_{j} \in I\left[q_{l}, a\right]$ If $M[i, j]=0$, let $M[i, j]=\operatorname{Min}\left\{f_{M}\left(q_{i}, w, q_{l}\right), f_{M}\left(q_{j}, w, q_{l}\right)\right\}$ for some $w \in \Sigma^{*}$ and append $\left(q_{i}, q_{j}\right)$ to NewPair.

Step5:

If $M[i, j]>0$ whenever $1 \leq i<j \leq n$, then $M$ is a directable fuzzy automaton, otherwise not.

Step 1:

Initialize Fuzzy Matrix $M$

$M[i, j]=\left(\begin{array}{cccc}0 & 0 & 0 & 0 \\ 0 & 0 & 0 & 0 \\ 0 & 0 & 0 & 0 \\ 0 & 0 & 0 & 0\end{array}\right)$

NewPair $=\{\epsilon\}$.

Step 2:

Inverted transition table $I$

$\begin{aligned} I\left[q_{1}, a\right] & =\{\phi\} & I\left[q_{1}, b\right] & =\left\{q_{2}, q_{3}\right\} \\ I\left[q_{2}, a\right] & =\{\phi\} & I\left[q_{2}, b\right] & =\left\{q_{4}\right\} \\ I\left[q_{3}, a\right] & =\left\{q_{1}, q_{3}, q_{4}\right\} & I\left[q_{3}, b\right] & =\left\{q_{1}\right\} \\ I\left[q_{4}, a\right] & =\left\{q_{2}\right\} & I\left[q_{4}, b\right] & =\{\phi\} .\end{aligned}$

Step 3:

From the above inverted transition table $I$,find all pairs such that $\left|I\left[q_{k}, a\right]\right|>1$.

$I\left[q_{3}, a\right]=\left\{q_{1}, q_{3}, q_{4}\right\} \quad I\left[q_{1}, b\right]=\left\{q_{2}, q_{3}\right\}$.

Now consider each pair $\left(q_{i}, q_{j}\right)$ with $i<j$, we get

$\left\{\left(q_{1}, q_{3}\right),\left(q_{1}, q_{4}\right),\left(q_{2}, q_{3}\right),\left(q_{3}, q_{4}\right)\right\}$.

$M[1,3]=\operatorname{Min}\left\{f_{M}\left(q_{1}, a, q_{3}\right), f_{M}\left(q_{3}, a, q_{3}\right)\right\}$ $=\operatorname{Min}\{0.7,0.3\}=0.3$.

$M[1,4]=\operatorname{Min}\left\{f_{M}\left(q_{1}, a, q_{3}\right), f_{M}\left(q_{4}, a, q_{3}\right)\right\}$ $=\operatorname{Min}\{0.7,0.5\}=0.5$.

$M[2,3]=\operatorname{Min}\left\{f_{M}\left(q_{2}, b, q_{1}\right), f_{M}\left(q_{3}, b, q_{1}\right)\right\}$ $=\operatorname{Min}\{0.6,0.1\}=0.1$.

$M[3,4]=\operatorname{Min}\left\{f_{M}\left(q_{3}, a, q_{3}\right), f_{M}\left(q_{4}, a, q_{3}\right)\right\}$ $=\operatorname{Min}\{0.3,0.5\}=0.3$.

Therefore, the fuzzy matrix and NewPair list becomes,

$M[i, j]=\left(\begin{array}{cccc}0 & 0 & 0.3 & 0.5 \\ 0 & 0 & 0.1 & 0 \\ 0 & 0 & 0 & 0.3 \\ 0 & 0 & 0 & 0\end{array}\right)$

NewPair $=\left\{\left(q_{1}, q_{3}\right),\left(q_{1}, q_{4}\right),\left(q_{2}, q_{3}\right),\left(q_{3}, q_{4}\right)\right\}$.

Step 4:

Delete the first pair from NewPair list. That is, delete the pair 
$\left(q_{1}, q_{3}\right)$.

From $I$ find all pairs $\left(q_{i}, q_{j}\right), i<j .\left\{q_{2}, q_{3}\right\} \in I\left[q_{1}, b\right]$ and $\left\{q_{1}\right\} \in I\left[q_{3}, b\right]$.

The required pairs are

$\left\{\left(q_{1}, q_{2}\right)\left(q_{1}, q_{3}\right)\right\}$. Already $M[1,3]>0$. Therefore, leave the pair $\left(q_{1}, q_{3}\right)$.

$M[1,2]=\operatorname{Min}\left\{f_{M}\left(q_{1}, a a, q_{3}\right), f_{M}\left(q_{2}, a a, q_{3}\right)\right\}$

$=\operatorname{Min}\{0.3,0.2\}=0.2$

Now add the pair $\left(q_{1}, q_{2}\right)$ to NewPair.

Therefore, the fuzzy matrix and NewPair list becomes,

$M[i, j]=\left(\begin{array}{cccc}0 & 0.2 & 0.3 & 0.5 \\ 0 & 0 & 0.1 & 0 \\ 0 & 0 & 0 & 0.3 \\ 0 & 0 & 0 & 0\end{array}\right)$

NewPair $=\left\{\left(q_{1}, q_{4}\right),\left(q_{2}, q_{3}\right),\left(q_{3}, q_{4}\right),\left(q_{1}, q_{2}\right)\right\}$.

Delete the pair $\left(q_{1}, q_{4}\right)$ in NewPair list and no such $\left(q_{i}, q_{j}\right)$ exists for the pair $\left(q_{1}, q_{4}\right)$ in $I$.

The fuzzy matrix and NewPair list becomes,

$M[i, j]=\left(\begin{array}{cccc}0 & 0.2 & 0.3 & 0.5 \\ 0 & 0 & 0.1 & 0 \\ 0 & 0 & 0 & 0.3 \\ 0 & 0 & 0 & 0\end{array}\right)$

NewPair $=\left\{\left(q_{2}, q_{3}\right),\left(q_{3}, q_{4}\right),\left(q_{1}, q_{2}\right)\right\}$.

Delete the pair $\left(q_{2}, q_{3}\right)$. From $I$ find all pairs $\left(q_{i}, q_{j}\right), i<j$.

$\left\{q_{4}\right\} \in I\left[q_{2}, b\right]$ and $\left\{q_{1}\right\} \in I\left[q_{3}, b\right]$. The required pairs are $\left\{\left(q_{1}, q_{4}\right)\right\}$.

Already, $M[1,4]>0$. Therefore, leave the pair.

The fuzzy matrix and NewPair list becomes,

$M[i, j]=\left(\begin{array}{cccc}0 & 0.2 & 0.3 & 0.5 \\ 0 & 0 & 0.1 & 0 \\ 0 & 0 & 0 & 0.3 \\ 0 & 0 & 0 & 0\end{array}\right)$

NewPair $=\left\{\left(q_{3}, q_{4}\right),\left(q_{1}, q_{2}\right)\right\}$.

Delete the pair $\left(q_{3}, q_{4}\right)$. From $I$ find all pairs $\left(q_{i}, q_{j}\right), i<j$.

$\left\{q_{1}, q_{3}, q_{4}\right\} \in I\left[q_{3}, a\right]$ and $\left\{q_{2}\right\} \in I\left[q_{4}, a\right]$. The required pairs are $\left\{\left(q_{1}, q_{2}\right),\left(q_{2}, q_{3}\right),\left(q_{2}, q_{4}\right)\right\}$.

Already, $M[1,2]>0$ and $M[2,3]>0$.

Therefore, leave the pairs $\left(q_{1}, q_{2}\right)$ and $\left(q_{2}, q_{3}\right)$.

Consider the pair $\left(q_{2}, q_{4}\right)$.

$M[2,4]=\operatorname{Min}\left\{f_{M}\left(q_{2}, a a, q_{3}\right), f_{M}\left(q_{4}, a a, q_{3}\right)\right\}$

$$
=\operatorname{Min}\{0.2,0.3\}=0.2 \text {. }
$$

Add the pair $\left(q_{2}, q_{4}\right)$ in the NewPair list.

Therefore, the fuzzy matrix and NewPair list becomes,

$M[i, j]=\left(\begin{array}{cccc}0 & 0.2 & 0.3 & 0.5 \\ 0 & 0 & 0.1 & 0.2 \\ 0 & 0 & 0 & 0.3 \\ 0 & 0 & 0 & 0\end{array}\right)$

NewPair $=\left\{\left(q_{1}, q_{2}\right),\left(q_{2}, q_{4}\right)\right\}$.

Delete the pair $\left(q_{1}, q_{2}\right)$. From $I$ find all pairs $\left(q_{i}, q_{j}\right), i<j$.

$\left\{q_{2}, q_{3}\right\} \in I\left[q_{1}, b\right]$ and $\left\{q_{4}\right\} \in I\left[q_{2}, b\right]$.

The required pairs are $\left\{\left(q_{2}, q_{4}\right),\left(q_{3}, q_{4}\right)\right\}$.

Already, $M[2,4]>0$ and $M[3,4]>0$.

Therefore, leave the pairs $\left(q_{2}, q_{4}\right)$ and $\left(q_{3}, q_{4}\right)$.

The fuzzy matrix and NewPair list becomes,
$M[i, j]=\left(\begin{array}{cccc}0 & 0.2 & 0.3 & 0.5 \\ 0 & 0 & 0.1 & 0.2 \\ 0 & 0 & 0 & 0.3 \\ 0 & 0 & 0 & 0\end{array}\right)$

NewPair $=\left\{\left(q_{2}, q_{4}\right)\right\}$.

Delete the last pair $\left(q_{2}, q_{4}\right)$. From NewPair list and no such $\left(q_{i}, q_{j}\right)$ exists for the pair $\left(q_{2}, q_{4}\right)$ in $I$.

Therefore, the fuzzy matrix and NewPair list becomes,

$M[i, j]=\left(\begin{array}{cccc}0 & 0.2 & 0.3 & 0.5 \\ 0 & 0 & 0.1 & 0.2 \\ 0 & 0 & 0 & 0.3 \\ 0 & 0 & 0 & 0\end{array}\right)$

NewPair $=\{\epsilon\}$.

Now all entries in upper part of $M[i, j]>0$.

Hence, the fuzzy automaton is directable.

\section{CONCLUSION}

In this paper, we introduce a method for testing the directability of a fuzzy automaton and provide an algorithm to find whether a fuzzy automaton is directable or not using the mergeability relation of the states. Also, we provide a necessary and sufficient condition for a fuzzy automaton to be directable and congruence of a fuzzy automaton to be directing.

\section{REFERENCES}

[1] Cao. Y., Chen. G., Kerre. E. 2011. Bisimulations for fuzzytransition systems, IEEE Transactions on Fuzzy Systems, no. 19: $540-552$.

[2] Cao. Y., Ezawa. Y. 2012. Nondeterministic fuzzy automata, Information Sciences, no. 191: 86-97.

[3] Doostfatemeh. M., Kremer. S. C. 2005. New directions in fuzzy automata, International Journal of Approximate Reasoning, no. 38: 175-214.

[4] Kandel. A., Lee. S. C. 1979. Fuzzy switching and automata theory applications, Edward Arnold Publishers Ltd. London.

[5] Karthikeyan. V., Rajasekar. M. 2011. Relation in fuzzy automata, Advances in Fuzzy Mathematics, 6 no. 1: 121-126.

[6] Karthikeyan. V., Rajasekar. M. 2012. Local necks of fuzzy automata, Advances in Theoretical and Applied Mathematics, 7 no. 2: 393-402

[7] Karthikeyan. V., Rajasekar. M. 2015. $\gamma$ - Synchronized fuzzy automata and their applications, Annals of Fuzzy Mathematics and Informatics, 10 no. 2: 331-342.

[8] Mordeson. J. N., Malik. D. S. 2002. Fuzzy automata and languages-theory and applications, Chapman \& Hall/ CRC Press.

[9] Santos. E. S. 1968. General formulation of sequential machines, Information and Control no. 12: 5-10.

[10] Wee. W. G. 1967. On generalizations of adaptive algorithm and application $O f$ the fuzzy sets concept to pattern classification, Ph.D Thesis Purude University.

[11] Zadeh. L. A. 1965. Fuzzy sets, Information and Control, no. 8: $338-353$

[12] Zimmermann. H. J. 1985. Fuzzy set theory and its applications, International Series in Management Science/ Operation Research, Kluwer- Nijhoff, Boston, MA. 